\title{
Observations at geosynchronous orbit of a persistent Pc5 geomagnetic pulsation and energetic electron flux modulations
}

\author{
T. E. Sarris ${ }^{1,2}$, T. M. Loto'aniu ${ }^{3}$, X. Li ${ }^{1}$, and H. J. Singer ${ }^{4}$ \\ ${ }^{1}$ Lab. for Atmospheric and Space Physics, Univ. of Colorado, Boulder, CO, USA \\ ${ }^{2}$ Demokritus University of Thrace, Xanthi, Greece \\ ${ }^{3}$ Dept. of Physics, University of Alberta, Edmonton, Canada \\ ${ }^{4}$ NOAA Space Environment Center, Boulder, CO, USA
}

Received: 20 November 2006 - Revised: 12 June 2007 - Accepted: 13 July 2007 - Published: 30 July 2007

\begin{abstract}
A long lasting narrow-band $(4-7 \mathrm{mHz})$ Pc5 fluctuation event at geosynchronous orbit is presented through measurements from GOES- 8 and GOES-10 and the response of energetic electrons with drift frequencies close to the narrow-band pulsation frequency is monitored through a spectral analysis of flux data from the LANL-SOPA energetic electron instrument. This analysis shows electron flux modulations at the magnetospheric pulsation's frequency as well as at various other frequencies in the Pc5 range, related to the particles' drift-frequencies and their harmonics. A drift resonance effect can be seen, with electron flux modulation becoming more evident in the energy channels of electrons with drift frequencies closer to the wave frequency; however no net increase or decrease in energetic electron flux is observed, indicating that the net energy transfer and transport of electrons is not significant. This Pc5 event has a long duration, being observed for more than a couple of days at geosynchronous orbit over several traversals of the two GOES satellites, and is localized in azimuthal extent. Spectral analysis shows that most of the power is in the transverse components. The frequency of the narrow-band event, as observed at geosynchronous orbit shifts during the time of the event from $7 \pm 0.5 \mathrm{mHz}$ to about $4 \pm 0.5 \mathrm{mHz}$. On the ground, CARISMA magnetometers record no distinct narrow-band fluctuation in the magnetic field, and neither does Geotail, which is traversing the outer magnetosphere a few $R_{E}$ further out from geosynchronous orbit, at the same UT and LT that GOES- 8 and -10 observe the pulsations, suggesting that that there is no connection to external fluctuations originating in the solar wind. An internal generation mechanism is suggested, such as could be provided by energetic ring current particles, even though conclusive evidence could not be provided for this particular event. Through a statistical study, it is found that this event belongs to a class of similar events,
\end{abstract}

Correspondence to: T. E. Sarris

(theodore.sarris@lasp.colorado.edu) occurring predominantly in the post-noon region in the inner magnetosphere.

Keywords. Magnetospheric physics (Energetic particles, trapped) - Space plasma physics (Wave-particle interactions; Waves and instabilities)

\section{Introduction}

Geomagnetic pulsations in the Pc5 range of frequencies are Ultra Low Frequency (ULF), large-scale oscillations in Earth's magnetic field. Such pulsations are often observed in the magnetosphere: some events show a single spectral peak (e.g. Kivelson and Chen, 1995; Ohtani et al., 1999), whereas other observations have reported simultaneous spectral peaks over a number of different frequencies (e.g. Ruohoniemi et al., 1991; Samson et al., 1991; Walker et al., 1992; Francia and Villante, 1997; Kepko et al., 2002). Their excitation mechanism has been associated with various magnetospheric processes, which are generally divided into external or "upstream" processes, related to the solar wind flow, and internal magnetospheric processes, in which ULF waves are generated by, for example, strong anisotropies in the ring current ion populations.

In one upstream model of externally generated pulsations, compressional variations in the bow shock caused by solar wind pressure variations propagate directly through the bow shock, magnetosheath, and magnetopause into the lower magnetosphere. In this description the compressional pulsations can reflect between an outer boundary and a turning point within the magnetosphere (possibly the magnetopause and plasmapause respectively), forming standing waves (e.g. Mann and Wright, 1995). In another upstream model, a velocity shear at the boundary between plasmas of different flow velocities, such as observed between the magnetosheath flow and the magnetopause, generates surface waves which

Published by Copernicus Publications on behalf of the European Geosciences Union. 
are toroidal Pc5 waves in nature. There are several observations that have attributed the pulsations to external oscillatory sources in the solar wind: for example, Sarafopoulos (1995) reported Pc5 polarization signatures in the lobes that are very similar to those in the solar wind; Ohtani et al. (1999) investigated a narrow-band event which showed the same oscillation frequency on the ground, at geosynchronous orbit and in the morning-side flank of the magnetosphere and attributed the excitation to a Kelvin-Helmholtz instability at the magnetopause; and Kepko et al. (2002) reported correlations between multiple discrete frequencies in the solar wind number density, dynamic pressure, and in the geosynchronous magnetic field: they concluded that variations in the solar wind preceded and drove the geosynchronous signatures.

ULF pulsations may also originate through a variety of internal magnetospheric processes. In these processes, the pulsations are produced by the interaction between standing wave modes and unstable ion populations in the ring current, which are produced by large positive gradients in its distribution function in energy and/or L-shell (Hasegawa, 1969; Southwood et al., 1969). Standing wave modes have been studied systematically by Takahashi et al. (1992), who used spacecraft and ground data to determine the generation mechanism for Giant Pulsations, which show many similarities to Pc5 pulsations. The pulsations produced by ion anisotropies are expected by theoretical estimates to have large azimuthal wave numbers $(m)$, to be heavily damped, and to remain close to the source region. Such high$m$ pulsations have been reported by, e.g., Misra et al. (1985), who used radar observations to determine the azimuthal mode number and phase velocity of the Pc5 waves. Such ULF pulsation signatures are expected to move westward, following the drift motion of the unstable ion population. These theoretical predictions were confirmed by Takahashi et al. (1985a), who showed multi-spacecraft observations of compressional Pc5 waves at geosynchronous orbit which have large $m$-values (between 40 and 120) and propagate westward. A theoretical explanation for the internal generation of magnetospheric pulsations has also been given by Vetoulis and Chen $(1994,1996)$, who placed the excitation area of the pulsations in a region in which there is a minimum in the poloidal mode frequency with respect to $\mathrm{L}$ and associated the location of this minimum with a minimum in pressure. Denton et al. (2003) tested whether this mechanism actually occurs, and found instead that the minimum in the poloidal mode frequency is associated with sudden decreases in plasma density, which typically occur in regions localized in L at the plasmapause. Lately, Ozeke and Mann (2001) developed a model which would result in favourable locations for the development of unstable ion distributions at the boundary between open and closed electron orbits; their model predicts the occurrence of localized storm-time Pc5 pulsations with monochromatic signatures, expected to appear mostly in the afternoon sector.
Early observations have shown that, during the time that Pc5 waves are observed, energetic particle flux is modulated by the waves (Lanzerotti et al., 1969; Kokubun et al., 1977). Theoretically, the relation between wave activity and energetic particle dynamics has been explored by radial diffusion theory, in which stochastic variations in the electric and magnetic fields result in diffusion of particles across drift shells through violation of the third adiabatic invariant (Falthammar, 1968; Schulz and Lanzerotti, 1974). By the radial inward or outward motion of particles across drift shells, flux changes (increases or decreases) could be observed by the "flattening" of a radial gradient of phase space density (outward or inward, respectively) (Green and Kivelson, 2004). Drift-resonant interactions between waves and energetic electrons have been demonstrated by MHD simulations, which have shown that in the magnetosphere ULF waves of frequency $\omega$ will preferentially accelerate electrons when the resonant condition $\omega=m \omega_{d}$ or $\omega=(m \pm 1) \omega_{d}$ is met (see, for example, Elkington et al., 1999), where $\omega_{d}$ is the electron drift frequency and $m$ is the azimuthal mode number of the fluctuation. However such interactions are generally difficult to observe in the magnetosphere, as most of the time multiple magnetospheric processes occur at the same time, affecting energetic particles in a complex way. In addition broadband ULF fluctuations affect the whole energy spectra of energetic particles simultaneously, making it hard to identify resonant interactions with particles having a particular drift frequency $\omega_{d}$.

In this study we present a narrow-band magnetospheric pulsation event that occurs during quiet magnetospheric conditions for a prolonged time, focusing on the spectral and temporal characteristics of the pulsation and on the interaction of the pulsation with energetic electrons. This magnetospheric pulsation is continuous with frequencies in the Pc5 range, which includes pulsations from 2 to $8 \mathrm{mHz}$, or 500 to $125 \mathrm{~s}$ periods (Jacobs et al., 1964). What is of particular interest is the long duration (several days) of the selected event, observed through multiple passages of the GOES geosynchronous satellites, and its spatial localization (few hours of local time in azimuth) in the dayside region. Over time, a shift in the frequency is observed at geosynchronous orbit. Through a spectral analysis of geosynchronous energetic electron flux, we monitor the energetic electron flux modulation caused by these pulsations, as observed by LANL satellites. By monitoring multiple LANL-SOPA electron energy channels we show evidence for a drift resonant interaction, with flux modulation being more intense for electrons whose drift frequencies are close to the frequency range of the pulsation event. However no net flux change is observed, which we attribute to a lack of sufficient gradient in phase space density. The narrow-band pulsation event is also monitored through CARISMA ground magnetic field and Geotail outer magnetosphere magnetic field measurements. The observations show that the pulsation is more likely tied to an internal generation mechanism, since there is no noticeable 
GOES-08, Feb. 16-19, 2001 (047-050)

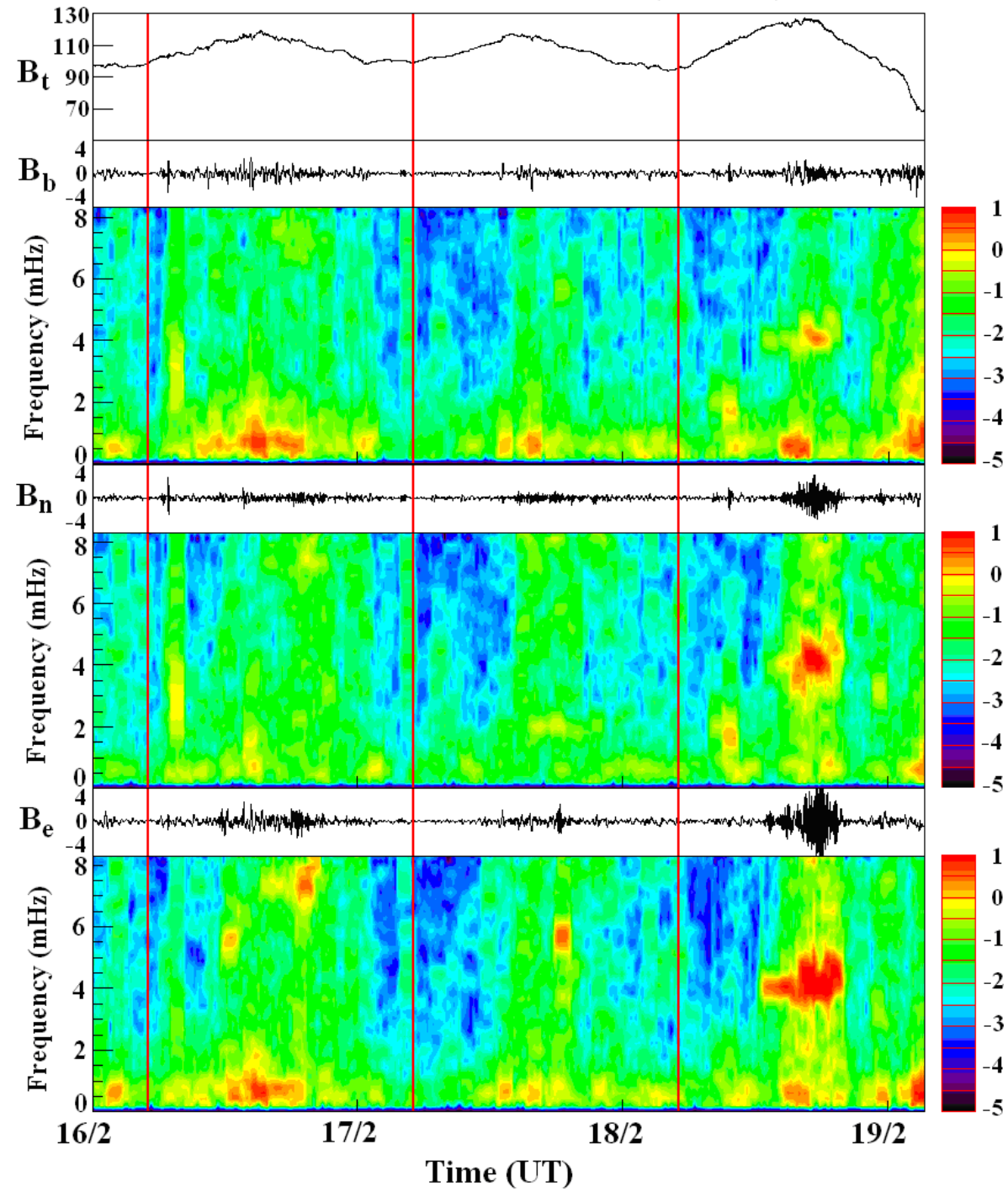

Fig. 1a. The magnetic field of the pulsation measured by GOES-8 from 16 to 18 February 2001 is plotted in the Mean Field-Aligned coordinate system. $B_{t}$ : total magnetic field, $B_{b}$ : field-aligned component, $B_{n}$ : normal - radially outward at the magnetic equator, and $B_{e}$ : eastward. The Dynamic Power Spectra of each component are plotted in color for frequencies up to $8 \mathrm{mHz}$ ( $\mathrm{Pc} 5 \mathrm{range}$ ). The color scale corresponds to the logarithm of the power, in $\mathrm{nT}^{2} / \mathrm{Hz}$. The red lines correspond to midnight in Local Time, or 05:00 UT.

narrow-band pulsation signature in the solar wind. There is also no noticeable signature on ground magnetometers for this event. A long term study of the occurrence distribution of similar narrow-band ULF pulsations is also presented, as observed during an 8-year interval by GOES-8 at geosynchronous orbit.

\section{Observations}

In this section the narrow-band fluctuation event is presented. In Sect. 2.1 the dynamic power spectra of magnetic field measurements from GOES-8 and GOES-10 geosynchronous satellites are shown over the course of 3 consecutive days. In Sect. 2.2 a statistical study of many similar events over an 8-year period is presented, focusing on the distribution of the events in local time and frequency. In Sect. 2.3 ground magnetometer measurements at the footprint of the geosynchronous satellites are presented. During the same period, Geotail was fortuitously traversing the dayside outer magnetosphere in the equatorial plane; its magnetic field measurements are presented in Sect. 2.4. Energetic electron measurements, available from LANL geosynchronous satellites, enable us to monitor the flux modulation caused by drift resonant interactions between energetic electrons and these fluctuations; these measurements are presented in Sect. 2.5. 


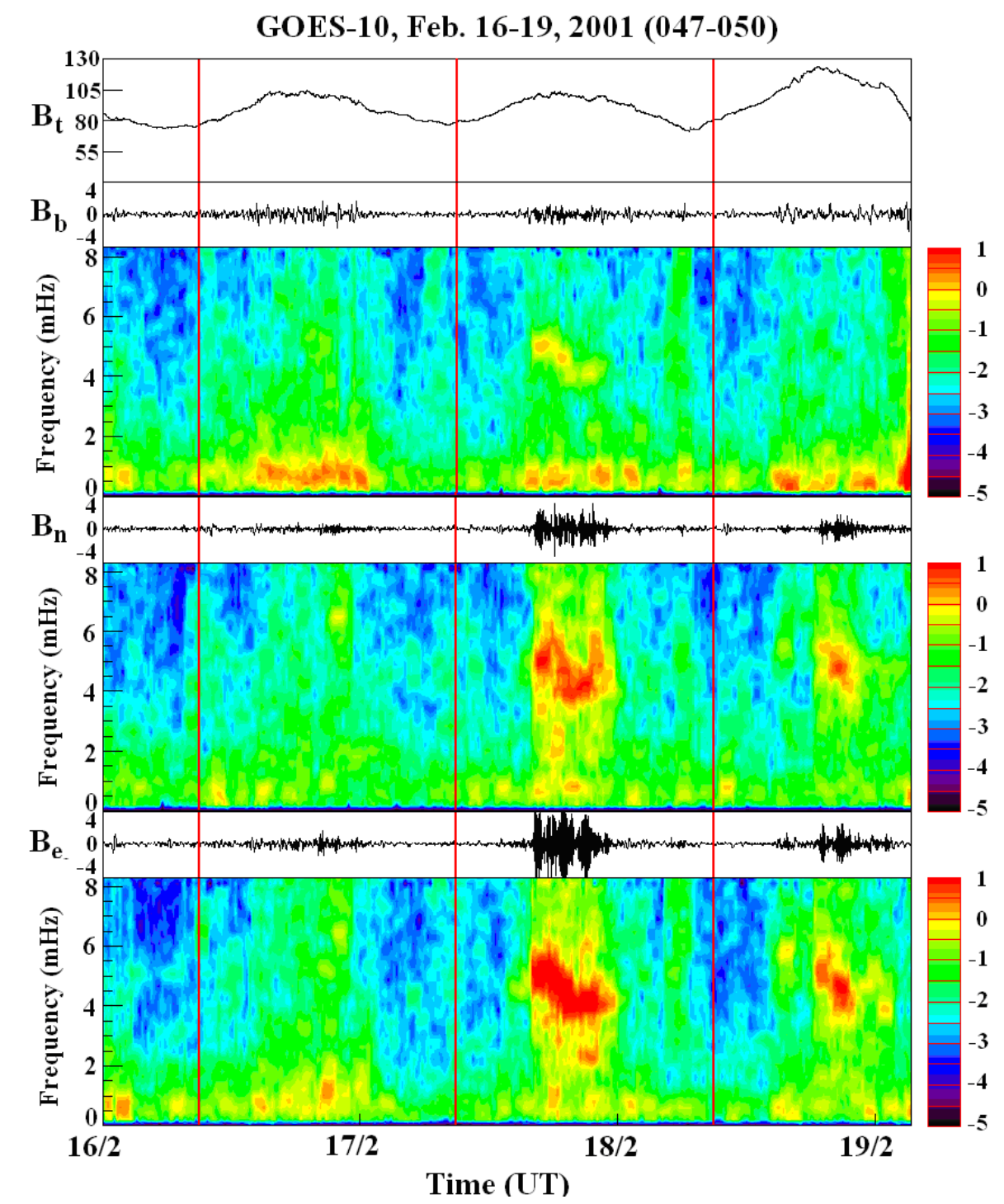

Fig. 1b. The magnetic field of the pulsation measured by GOES-10 from 16 to 18 February 2001 is plotted in the Mean Field-Aligned coordinate system, similar to Fig. 1a. The red lines correspond to the time when GOES-10 crosses local midnight, which is at 09:00 UT every day.

\subsection{Dynamic power spectra of the fluctuations}

The magnetic field measurements and their Dynamic Power Spectra (DPS) over the three-day period that the fluctuations were observed, from 16 to 18 February 2001, are plotted in Figs. 1a and $\mathrm{b}$ using 1-min time resolution measurements from satellites GOES-8 and GOES-10 respectively (Singer et al., 1996). GOES-8 was located at geomagnetic longitude $75^{\circ}$ West and GOES-10 at $135^{\circ}$ West, at geosynchronous orbit. In order to separate the ULF field variations perpendicular to as well as along the magnetic field direction, the components of the magnetic field vector are projected in the Mean Field-Aligned (MFA) coordinate system. The unit vectors of the MFA coordinate system are denoted $\hat{\boldsymbol{b}}, \hat{\boldsymbol{n}}$, and $\hat{\boldsymbol{e}}$, in which $\hat{b}$ is the direction of the mean magnetic field, obtained from a 30-min running average of the instantaneous magnetic field direction, $\hat{\boldsymbol{e}}$ is perpendicular to both the radius from the Earth's center and to $\hat{\boldsymbol{b}}$, pointing eastward, and $\hat{\boldsymbol{n}}$ completes the orthogonal system, pointing radially outward at the magnetic equator. The average magnetic field was subtracted from the projection of the magnetic field onto the average unit vector $\hat{\boldsymbol{b}}$. The total magnetic field, $B_{t}$ is plotted in the top panel, whereas below are plotted the $B_{b}, B_{n}$ and $B_{e}$ components (black and white plots) and their corresponding DPS (color plots), as denoted in each figure. The DPS calculations were performed by sliding an 80-min wide Hanning window through the data and performing an FFT on the subset of the signal within the window. For a one-minute sampling 
frequency, the Nyquist frequency (and hence the maximum frequency we can monitor in this data set) is $8.3 \mathrm{mHz}$. The frequency resolution in this analysis is $0.2 \mathrm{mHz}$.

This particular narrow-band fluctuation event occurred during a 3-day period of very quiet magnetospheric conditions: the Kyoto $D_{s t}$ index during this time ranged between -10 and 0 , recovering from a $D_{s t}$ value of -50 on $\sim 00: 00$ UT of 14 February, 2 days prior to the time-period of interest. The successive passages of GOES-8 and GOES-10 satellites on each day provides a unique opportunity to study the duration and azimuthal extent of the narrow-band fluctuations. On 16 February, both GOES-8 and GOES-10 observe a faint, low-power narrow-band fluctuation signature mainly in the $B_{e}$ but also in the $B_{n}$ components while they cross the dayside region, at a narrow band of frequencies around $\sim 7.5 \pm 0.5 \mathrm{mHz}$ at GOES- 8 and $\sim 6.5 \pm 0.5$ at GOES-10. One possible reason for that is the fact that the two geosynchronous satellites traverse different L-shells, as described by Onsager et al. (2004), thus monitoring plasma populations with different densities; another possible reason is that the two s/c monitor plasmas with an azimuthal gradient in mass density, or that the frequency changes in time. This is further discussed in Sect. 3. There is a narrow-band pulsation at $\sim 5-6 \mathrm{mHz}$ earlier on the 16th that occurs at a pre-noon local time; we believe that it is unlikely that this is related to the event studied. On 17 February the narrow-band fluctuation can still be seen with the same intensity in the $B_{e}$ component, while its frequency has dropped to $\sim 5.5 \pm 0.5 \mathrm{mHz}$; five hours later, by the time GOES-10 crosses the same region, the narrow-band fluctuation has intensified. The azimuthal extent of the pulsations at this time is found to be about $7 \mathrm{~h}$ of local time, as they are observed from 18:00 UT, when GOES-10 is located at about 09:00 LT, until 01:00 UT of 18 February when GOES-10 is located at 16:00 LT. The frequency of the narrow-band fluctuation event on this passage gradually shifts from $5.0 \pm 1.0 \mathrm{mHz}$ at the beginning of the event to $4.0 \pm 1.0 \mathrm{mHz}$. An intense narrow band fluctuation is also observed at the time of the next passage of GOES-8, on 18 February (from 19:00 UT until 22:00 UT corresponding to $14: 00$ LT to $17: 00 \mathrm{LT}$ ), and also at the time of the next passage of GOES-10 (from 18:00 UT until 22:30 UT corresponding to 09:00 LT to 13:30 LT), leading to the assumption that it is the same phenomenon that continues. A few hours later, by the time GOES- 8 crosses the same region again, the narrow-band fluctuation has disappeared, at least on the L-shells that GOES-8 and GOES-10 are monitoring. The pulsation is very faint in the DPS of the total magnetic field for both spacecraft. The immediate implication of the above observations is that the narrow-band fluctuation is noncompressional and is occurring mostly in the transverse components; furthermore, it is a localized event, with distinct and localized spatial (azimuthal) extent, rather than a more global oscillation with distinct and short temporal duration.

As it can be seen in Figs. 1a and b, there is a time period on 18 February over which the pulsations are observed si-
140 Events in $1995-2003$

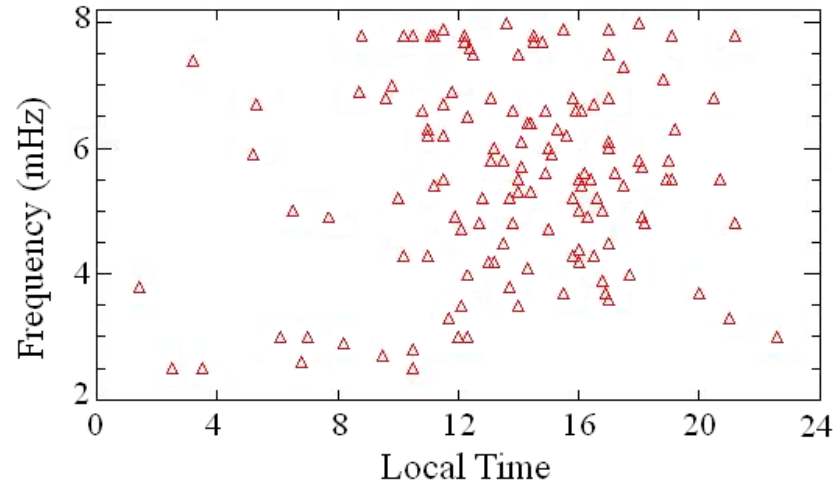

Fig. 2. Distribution in frequency ( 2 to $8 \mathrm{mHz}$ ) and local time of the narrow-band Pc5 pulsation events from GOES-8 geosynchronous measurements in the period from 1995 to 2003.

multaneously by both GOES-8 and GOES-10 (20:00 UT until 21:30 UT). During this time, the 4-h local time separation between the two GOES satellites would allow waves of mode numbers $m<6(=24 \mathrm{~h} / 4 \mathrm{~h})$ to be observed with a coherent shift in phase throughout the time that both satellites observe the pulsations simultaneously. In order to investigate if there are such periods of consistent phase difference we performed a comparison between the phases (cross-phase spectrogram) of GOES-8 and GOES-10 magnetic field on 18 and 19 February, when both spacecraft were in the active region. This calculation has shown no cross-correlation between the two signals (plot of cross-phase spectrogram not shown here), indicating that mode number $m$ should be higher than 6 .

\subsection{Distribution of narrow-band fluctuations}

A survey of the Dynamic Power Spectra of variations in the total field strength from GOES-8 measurements from 1995 to 2003 has yielded a total of 140 events of azimuthally (or temporally) localized narrow-band fluctuations in the total magnetic field. The distribution in local time and frequency of these events is given in Fig. 2. In this figure, each triangle marks the local time and frequency of the peak in power of the fluctuation, regardless of the local time extent and bandwidth of the fluctuations. The events were selected as narrow-band fluctuations if the power of the fluctuations at any frequency in the range 2 to $8 \mathrm{mHz}$ exceeded the power of the low-frequency background noise by at least a factor of 5. This factor was arbitrarily selected; however for most events the power at the peak of the narrow-band fluctuation exceeded the power of the background noise by at least an order of magnitude. The events varied among themselves in intensity, azimuthal/temporal extent and bandwidth; compared to all the narrow-band fluctuation events shown in this figure, the event presented in Fig. 1 could be described as moderately active. The particular event was selected as a 


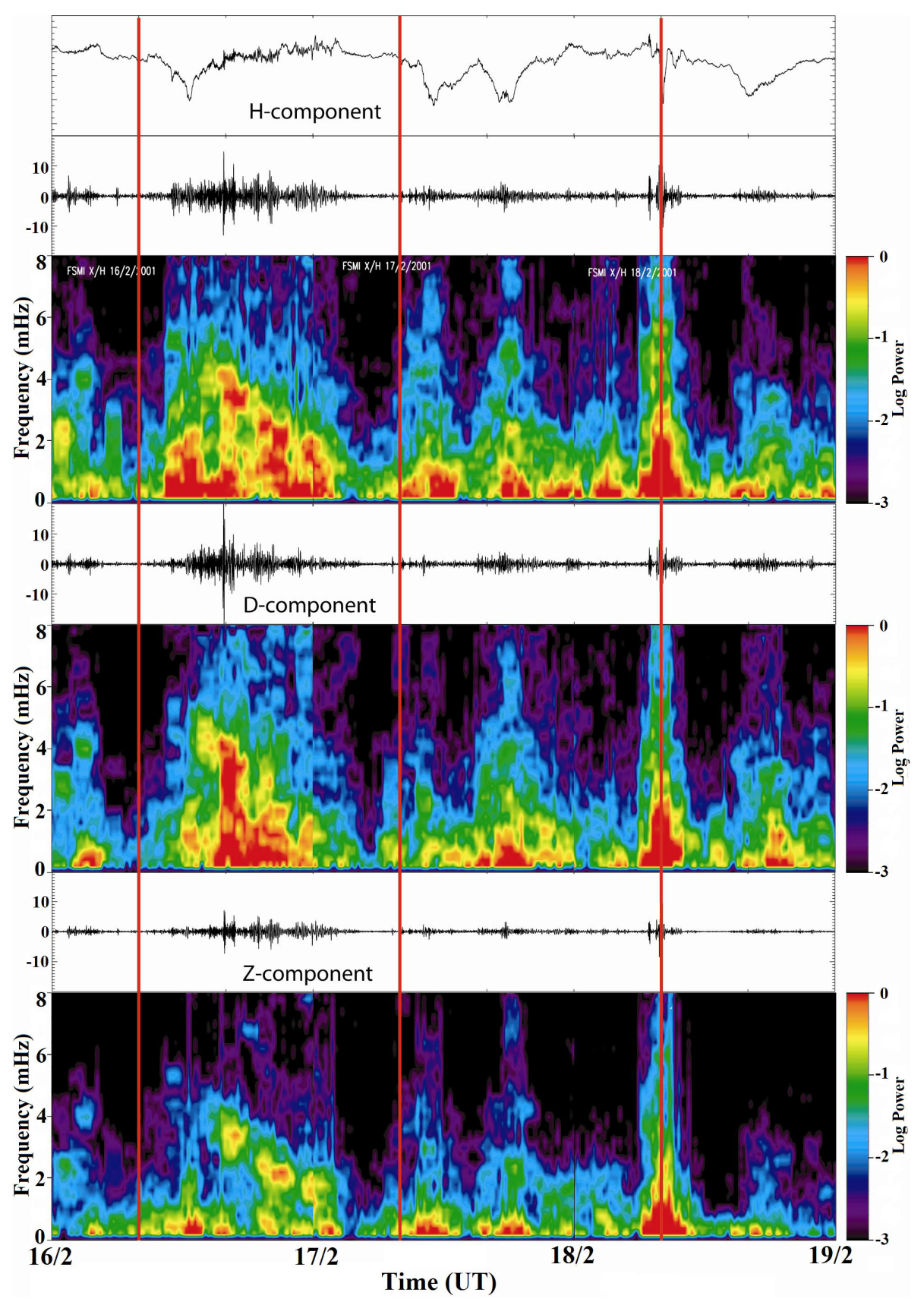

Fig. 3. Ground magnetometer measurements from station Fort Smith $\left(67.45^{\circ}\right.$ geomagnetic latitude, $306.15^{\circ}$ longitude and $\left.\mathrm{L}=6.84\right)$, close to the footprint of GOES-10 ( $66^{\circ}$ latitude, $296^{\circ}$ longitude). Local midnight on each day is shown with the red lines.

case study because of the duration of the event, as well as because of the quiet magnetospheric conditions during the three-day period around the pulsations, which enabled the study of the interaction of the pulsations with the energetic electron population that is presented below in Sect. 2.5.

From the distribution of events that is shown in Fig. 2, there is a clear preference for the post-noon region for the narrow-band pulsations. In previous studies, the occurrence of pulsations in the post-noon region has been related to in- ternal processes of the magnetosphere, such as kinetic instabilities of ring current ions and high beta plasma clouds (Anderson, 1993; Ohtani et al., 1999); also, pulsations in the post-noon region have been associated phenomenologically with poloidal wave modes, which are more commonly radially polarized (e.g. Arthur and McPherron, 1981; Engebretson et al., 1992; Anderson, 1993). The occurrence distribution and characteristics of the pulsations in the post-noon region are further discussed in Sect. 3. 


\subsection{Ground measurements}

For the present event, ground magnetic fluctuation signatures were acquired from the Canadian Array for Real-time Investigations of Magnetic Activity, CARISMA, formerly known as the CANOPUS magnetometer array (Samson et al., 1991). The ground magnetometer closest to the magnetic "footprint" of GOES-10 (66 ${ }^{\circ}$ geomagnetic latitude and $296^{\circ}$ geomagnetic longitude) is located at station Fort Smith $\left(67.45^{\circ}\right.$ geomagnetic latitude, $306.15^{\circ}$ longitude and $\left.\mathrm{L}=6.84\right)$; its measurements are presented in Fig. 3, using the same spectral analysis technique described above. In these figures, three days of ground magnetic field measurements around the local time of the narrow band fluctuation are plotted, corresponding to the same time-period as in Fig. 1. The plots correspond from top to bottom to the magnetic $\mathrm{H}, \mathrm{D}$, and $\mathrm{Z}$ components in geomagnetic coordinates, where $\mathrm{H}$ points in the horizontal magnetic north-south direction, D points east-west, with eastward positive, and $\mathrm{Z}$ points vertically downward into the Earth. The red lines correspond to the times that the ground station crosses local midnight.

From these plots it can be seen that there are significant broad-band ULF fluctuations in the morning-noon region on 16 February. These fluctuations gradually decrease on the 17 th and 18th, at the time that the narrow band pulsations at the GOES-10 location are intensifying. Narrow-band fluctuations can be distinguished in the Z-component; around the noon region, narrow-band fluctuations can be identified at $\sim 6.8 \mathrm{mHz}$ on 16 February, at $\sim 4.2 \mathrm{mHz}$ on the 17 th and at $\sim 2.4-3.0 \mathrm{mHz}$ on the $18 \mathrm{th}$. However they cannot be uniquely identified and associated with the strong fluctuations that are observed at geosynchronous orbit during these days. Possible reasons that inhibit waves in space from being observed on the ground are discussed in Sect. 3. Measurements from stations on the Churchill line of ground magnetometers, which are aligned close to the geomagnetic longitude of GOES-8, also show a weak correlation with the geosynchronous measurements.

\subsection{Outer magnetosphere measurements}

On the days that GOES-8 observed the narrow-band fluctuations, Geotail was fortuitously traversing the dayside outer magnetosphere in the equatorial plane. The orbit of Geotail is plotted in Fig. 4, together with the orbits of GOES-8 and GOES -10, in the X-Y plane (left panel) and X-Z plane (right panel), in GSE coordinates. Small triangles mark the spacecrafts' locations every hour. The locations of Geotail at 00:00 UT 17, 18 and 19 February are marked with asterisks. GOES- 8 and GOES-10 cross the midnight meridian (00:00 LT) at 05:00 UT and 09:00 UT, respectively, each day; their locations at 00:00 UT every day are also marked with asterisks.

The magnetic field measurements from the Geotail spacecraft are plotted in Fig. 5 with 64-s resolution in the same
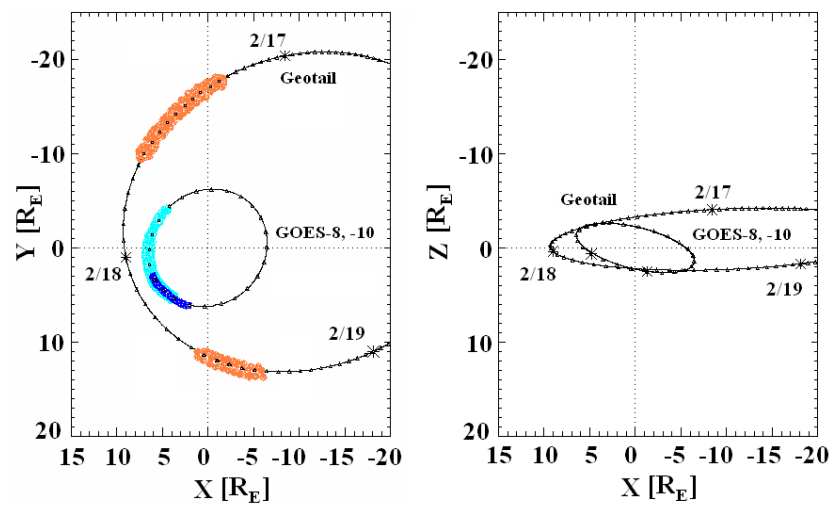

Fig. 4. The orbits of Geotail, GOES-8 and GOES-10 are plotted on the X-Y (left) and X-Z (right) planes in GSE coordinates for the period from 17 to 19 February 2001. The asterisks and triangles mark the location of the satellites at 00:00 UT and at every hour in UT, respectively. The orange-shaded areas indicate the regions where Geotail observes intense ULF fluctuations; the overlapping regions marked in blue and cyan mark the regions where narrow band fluctuations are recorded by GOES- 8 and GOES-10 respectively on 17 and 18 February.

format as in Fig. 1, i.e. the total magnetic field $\left(B_{t}\right)$ and the three components of the magnetic field in the MFA coordinate system $\left(B_{b}, B_{n}\right.$ and $\left.B_{e}\right)$ are plotted in black and white as marked, and their corresponding DPS are plotted below in the color panels; the DPS were calculated in a similar manner as described for the GOES magnetic field measurements. During the two-day passage of Geotail through the dayside magnetosphere there are two distinct time intervals of intense ULF fluctuations, marked in orange in the three MFA components of the magnetic field in Fig. 5; the spatial extent over which these fluctuations were observed are also marked in orange in Fig. 4. From the location of the orange areas we can conclude that these intense fluctuations correspond to Geotail crossings of the magnetopause-magnetosheath: it is well established that in the region which forms between the solar wind and terrestrial regimes, earthward of the bow shock, the magnetic field is usually highly disturbed, as the shear in the flow across the magnetopause leads to the formation of instabilities such as the Kelvin-Helmholtz instability (e.g. $\mathrm{Wu}, 1986)$. The observations by Geotail are consistent with this picture, showing intense fluctuations in the morning and afternoon flanks, where the shear in the flow gets bigger and hence the disturbance in the magnetic field is stronger.

From the Geotail orbit and the local time occurrence of the pulsations that is extracted from Fig. 1, it can be seen that at 00:00 UT 18 February (the time of most intense narrow-band field fluctuations, according to Fig. 1) Geotail would be in an ideal location to measure narrow-band compressional pulsations, if these were extending just a few $R_{E}$ further out from geosynchronous orbit, or if they were related to externally produced oscillations of the bow shock caused by solar wind 


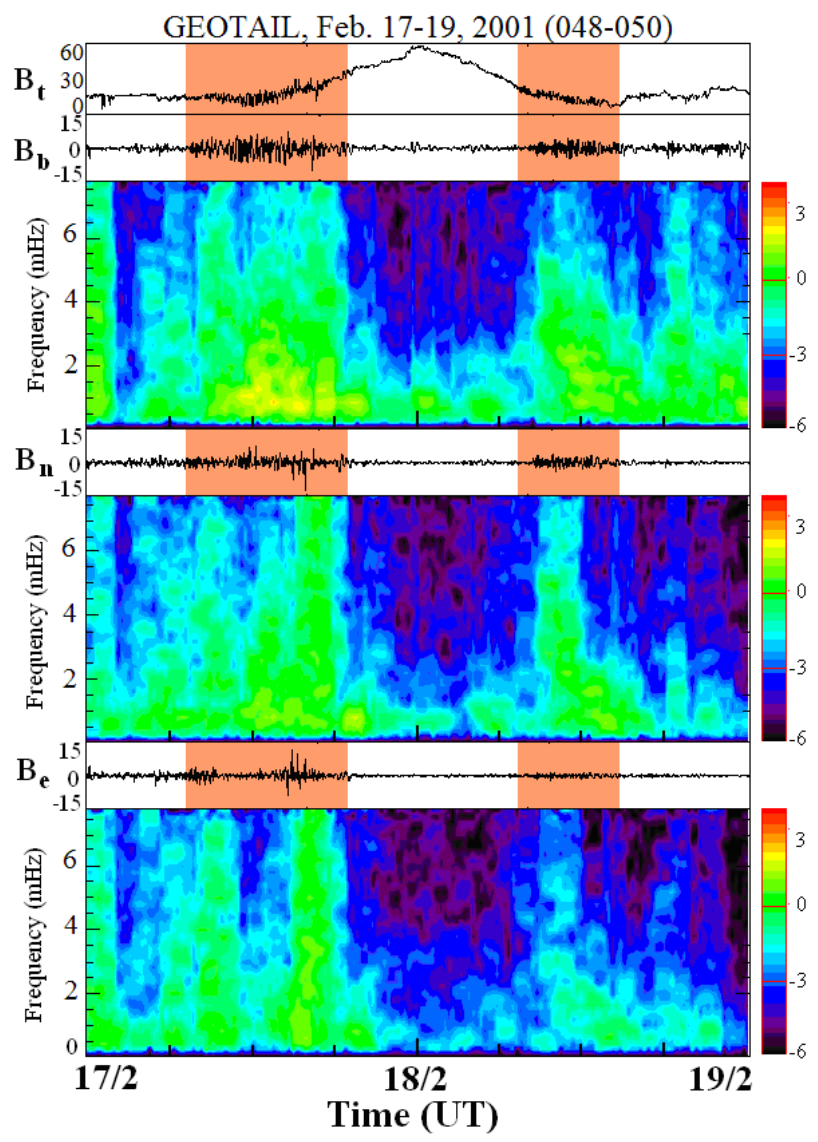

Fig. 5. A sequence of 2 days of Geotail magnetometer measurements, from 17 to 19 February 2001. The total magnetic field and the three components in the Magnetic Field Aligned coordinate system are plotted in black \& white, in nT, as marked; the corresponding Dynamic Power Spectra are plotted below in color, in $\mathrm{nT}^{2} / \mathrm{Hz}$. The two orange-shaded time periods in each MFA component mark the regions of intense ULF fluctuations in Geotail data; the corresponding extent along Geotail's orbit is also marked in orange in Fig. 6.

pressure pulses; such pulsations would be expected to propagate through the shock, sheath, and magnetopause into the lower magnetosphere, carrying similar frequency signatures throughout the whole region. However no particular fluctuations are detected in the noon or post-noon region in Fig. 5 that could be associated with the narrow-band fluctuations in the corresponding regions at geosynchronous orbit.

\subsection{Energetic electron flux measurements}

The narrow-band fluctuation on 18 February has its peak amplitude at $4 \mathrm{mHz}$, corresponding to a $250 \mathrm{~s}$ period; this pulsation period is of the order of the gradient-B drift-period of $3 \mathrm{MeV}$ electrons at geosynchronous orbit. Thus it is expected that the narrow-band fluctuations will resonantly interact with $3 \mathrm{MeV}$ electrons. In order to monitor the na- ture and the effects of this interaction, we examine energetic electron flux measurements from four different energy channels of the LANL Synchronous Orbit Particle Analyzer (SOPA) instrument on the geosynchronous satellites maintained by the Los Alamos National Laboratory (Belian et al., 1992; Reeves et al., 1997). Measurements from satellite LANL 1989-046 on 18 February are presented in Fig. 6 for four selected differential energy channels. The time resolution of the measurements is $1 \mathrm{~min}$ and the energy ranges for the four channels are: $0.5-0.75 \mathrm{MeV}, 0.75-1.1 \mathrm{MeV}, 1.8-$ $3.5 \mathrm{MeV}$, and $3.5-6.0 \mathrm{MeV}$. Electron flux measurements for each of the four energy channels are given in the upper (black $\&$ white) panel, while in the lower (color) panel we plot the corresponding dynamic spectra of the flux measurements. In this analysis we treat the 1-min-resolution flux measurements in a fashion similar to the spectral analysis performed for the magnetic field measurements described in Sect. 2.1 in order to extract flux modulation signatures; the yellow vertical line in each plot indicates the time when LANL 1989-046 crosses the local noon meridian. In order to investigate any drift resonant effects, the range of drift frequencies of the electrons within each energy channel were calculated numerically; the upper and lower drift frequencies for each channel are plotted as red horizontal lines at the corresponding frequencies on the plots of the dynamic spectra of the flux fluctuations. It should be noted however that, due to the exponential drop of particle flux as their energy increases, electrons towards the lower end of the energy range of a detector are expected to be more abundant, and are thus expected to dominate the behavior of the population within the energy channel.

In Fig. 6a it can be seen that there is more intense flux modulation around the post-noon regions, hence around the local time that the narrow-band fluctuation is recorded by the GOES spacecraft, which was 14:00-17:00 LT. In particular, there is a distinguishable flux modulation at $4 \mathrm{mHz}$, i.e. at the frequency of the narrow-band pulsation. This is expected, since the narrow-band pulsation will cause a local fluctuation of the magnetic field at that location: electrons gradient-B drifting through this region will be forced to move radially inward and outward at the frequency of the fluctuation while they follow constant-B contours, which appears as a flux modulation at the same frequency. The range of electron drift frequencies within this energy channel is 1.0 to $1.4 \mathrm{mHz}$. It can be seen that there is also enhanced flux modulation close to the drift frequencies of electrons in this energy channel: this effect is also expected, since any non-adiabatic disturbance in the magnetic field (in this case, any disturbance on a time scale much shorter than the electrons' drift period) will cause a local disturbance in the electron population, which will drift-echo around the earth, and which will appear as a flux modulation at the electrons' drift frequency. In Fig. $6 \mathrm{~b}$ the range of drift frequencies is 1.4 to $1.9 \mathrm{mHz}$, with most of the electrons drifting closer to $1.4 \mathrm{mHz}$, as described above; the flux modulation at $4 \mathrm{mHz}$ as well as at the electrons' drift frequencies appears again, 

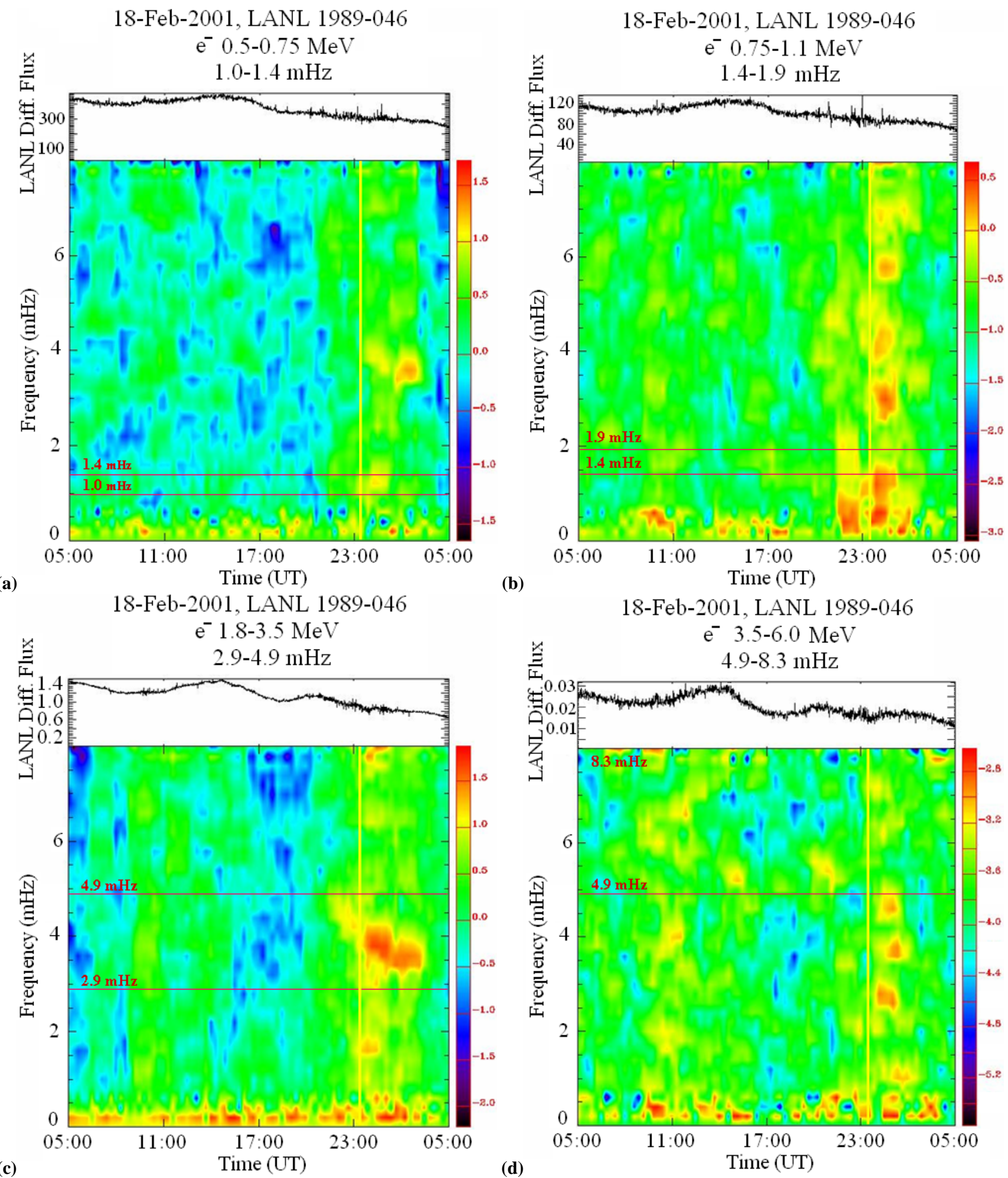

Fig. 6. Dynamic Spectra of the flux modulation of electrons measured by the SOPA instrument onboard LANL 1989-046 geosynchronous satellite on 18 February 2001 from four different energy channels. Distinct flux modulation is observed around the local time of the narrowband magnetic field fluctuations. The red lines on these plots mark the drift-frequencies of electrons at the lower and upper ends of each energy channel and the yellow line marks the time when the spacecraft crosses local noon. 
however there are fluctuations at other isolated frequencies as well. A careful examination shows that, excluding the modulation at $4 \mathrm{mHz}$, the other modulations appear at $\sim 3 \mathrm{mHz}$ and $\sim 6 \mathrm{mHz}$, which corresponds to approximately twice and four times the electrons' drift frequency. Electrons in the energy range shown in Fig. 6c have drift frequencies within 2.9 to $4.9 \mathrm{mHz}$ that encompass the $4 \mathrm{mHz}$ narrow-band fluctuation; the flux modulation in this case is concentrated on a single dominant frequency that shows the most intense flux modulation. In Fig. 6d, which refers to higher energy electrons and drift frequencies 4.9 to $8.3 \mathrm{mHz}$, higher than the narrow-band magnetospheric frequency, the flux modulation is weaker and occurs again at several sub-harmonics. All other LANL SOPA energy channels were similarly analyzed and a consistent picture was observed, with the flux modulation signatures becoming less intense the further the driftfrequencies of the electrons within each channel deviate from the narrow-band pulsation's frequency.

\section{Discussion}

Following is a discussion of the various features of the narrow-band fluctuation that was observed at geosynchronous orbit, as well as the 8-year statistical survey of similar pulsations.

3.1 On the nature and excitation mechanism of the pulsation

As discussed by Takahashi et al. (1985b) and Li et al. (2006), a factor that might determine the wave excitation is the position of the plasmapause, which is known to expand during times of reduced geomagnetic activity (Chappell et al., 1970). Transverse Pc4 and Pc5 pulsations have been observed near the plasmapause (Singer et al., 1982) as well as near detached density enhancements (Lanzerotti et al., 1975). The post-noon preference in the occurrence distribution of the observed narrow-band events shown in Fig. 2 might be related to the formation of plasma tails or drainage plumes that often form in the dusk sector and can either remain fairly stationary in local time or "wrap" around the plasmasphere as it corotates toward midnight (e.g. Chen et al., 1976).

A study of the LANL-MPA plasma instrument onboard the LANL 1989-046 satellite has shown (although not presented here) an increase in plasma number density by about $50 \%$ in the $1 \mathrm{eV} / \mathrm{e}$ to $130 \mathrm{eV} / \mathrm{e}$ ion channel around the local time of the Pc5 pulsations; a spectral analysis carried out in a similar fashion to that of the LANL-SOPA data, as described in Sect. 2.5, has shown a density modulation at $4 \mathrm{mHz}$. The higher energy ion channel, $130 \mathrm{eV} / \mathrm{e}$ to $45 \mathrm{keV} / \mathrm{e}$, and the electron channel, $30 \mathrm{eV}-45 \mathrm{keV}$, show no response to the magnetic field fluctuation; however, we note that this energy range is very broad and its behavior will be determined mostly by the lower part of the energy spectrum, so that any populations in the keV-range with sufficient amounts of free energy to resonantly interact via bounce-motion resonance with the fluctuating magnetic field would be averaged out. Thus better energy resolution of the low-energy plasma would allow us to address the causal mechanism of these pulsations with greater certainty.

It has been suggested that unstable distributions can be generated by the splitting and divergence of adjacent ion orbits (Cowley and Ashour-Abdalla, 1976; Hughes et al., 1978; Ozeke and Mann, 2001), favorably at the boundary between open and closed ion orbits. What is of particular interest in this model is that it predicts the occurrence of localized storm-time Pc5 pulsations with monochromatic signatures, most notably in the afternoon sector, making it a very likely candidate mechanism for this event. A detailed study of the ion distribution and the amount of free energy contained, together with a simulation that would reveal the boundary between open and closed particle orbits for the conditions on the days of this event could identify the exact excitation mechanism and energy source for these pulsations.

\subsection{On the frequency signatures of the pulsation}

An interesting feature of this event is the drop in frequency that is observed in Fig. 1 from 16 February to 18 February, which is particularly evident in GOES-8 data. If these pulsations were cavity mode oscillations between the magnetopause and plasmapause, then an increase of the cavity size following the relaxation of the magnetosphere after a storm could lead to such a frequency drop. As both the magnetopause and plasmapause are known to expand in the recovery phase after a storm, this would mean that the magnetopause expands faster than the plasmapause. On the other hand, if these pulsations are of internal origin, resulting from wave-particle interactions between resonating field lines and plasmas of a particular energy in a localized area of the plasmasphere or plasmapause, then a repopulation of the plasmasphere and the subsequent increase in density would similarly lead to a decrease of frequency.

From Fig. 1 it can be seen that there is a difference in the frequency of the narrow-band fluctuation between the two satellites, with GOES-10 consistently recording a lower frequency than GOES-8. One possible reason is the fact that the two geosynchronous satellites traverse different Lshells. This effect has been described in detail by Onsager et al. (2004): although both spacecraft are located at the geographic equator, the Earth's magnetic dipole is tilted toward the geographic equator in approximately the longitude of GOES-8. This has the effect that GOES-10 will be located at smaller geomagnetic latitudes than GOES- 8 and will be measuring particles and fields at lower L than GOES-8, thus monitoring different plasma populations. The frequency of oscillation for Alfvén wave mode forms is proportional to the magnetic field strength and inversely proportional to the effective magnetic field line length and plasma density 
(Dungey, 1954); however, the Alfvén velocity is at minimum near the equatorial plane, close to which both spacecraft are located, and thus the field line resonant frequency will be mostly affected by plasma density (Waters et al., 1996). As, in general, plasma density around the equatorial plane will increase with decreasing $\mathrm{L}$, the innermost satellite is expected to record lower frequencies. To investigate if this is a reasonable assumption we consider the following:

Field line eigenfrequency in the magnetosphere is proportional to the Alfvén velocity and inversely proportional to the field line length, $l$, which is approximately proportional to $\mathrm{L}$. Thus it varies as $B_{e q} / L n^{1 / 2}$ where $B_{e q}$ is the equatorial value of the magnetic field strength and $n$ is the equatorial value of the mass density. Since $B_{e q}$ decreases with $L$ as $L^{-3}$ in a dipole field, $n$ must decrease faster than $L^{-8}$ for eigenfrequency to increase with $L$. A density gradient this sharp could be observed at the plasmapause, however the LANL MPA measurements described above are not showing indications of a plasmapause crossing. Other possible reasons are either a change of the frequency with time, as GOES-8 and GOES-10 observe the waves at different UT, or perhaps an azimuthal gradient in plasma mass density; however more measurements are needed to conclude the exact cause of the frequency difference between GOES-8 and GOES-10.

\subsection{On the lack of narrow-band signatures on the ground}

Narrow-band fluctuation signatures detected at the same time on the ground and in the magnetosphere have been reported in the past (e.g. Kivelson and Chen, 1995; Ohtani et al., 1999); however for this particular event, as shown in Fig. 3, there are no distinct narrow-band signatures in ground measurements. The fact that some pulsations do not reach the ground has been discussed in many studies, which point out that ULF waves are modified in a very complex way by both ionospheric and ground currents along their propagation from the equatorial regions through the ionosphere to the ground (e.g. McPherron et al., 1972, and references therein). The ionosphere affects particularly high-m magnetospheric ULF waves, rotating, reflecting and/or attenuating the magnetic signatures of the waves as detected on the ground (e.g. Hughes and Southwood, 1976). Thus ground magnetometer data are far from optimal for the study of high-m ULF waves. The lack of definite ground signatures is in fact reinforcing the hypothesis that the observed Pc5 pulsations are high-m, internally generated Alfvénic waves.

\subsection{On the distribution of pulsations in local time}

The 8-year statistical study of narrow-band pulsations at geosynchronous orbit that was presented in Fig. 2 found a distribution of compressional narrow-band pulsations that was skewed towards the post-noon/evening sector, showing a different behaviour between the morning and evening side magnetosphere. What is of particular interest is the fact that, in the pre-noon region there are a large number of events with frequencies $f<3 \mathrm{mHz}$, whereas after noon there are essentially no events with $f<3 \mathrm{mHz}$; an explanation for this asymmetry cannot be provided at this point. Past statistical studies of the distribution of geomagnetic pulsations using smaller statistical samples have found that morning and afternoon pulsations vary significantly. These studies have shown that morning-side Pc5 waves are mostly standing Alfvén waves with a fundamental odd mode as their dominant mode of oscillation (Singer and Kivelson, 1979), even though harmonics of higher frequencies have also been reported; such pulsations are more commonly transverse to the direction of the magnetic field, polarized in the azimuthal direction (Takahashi and McPherron, 1984; Anderson et al., 1990), and have been attributed to external excitation mechanisms, and in particular the Kelvin-Helmholtz instability on the morning flank of the magnetosphere. On the other hand, pulsations in the post-noon and evening sector have been found to be more often related to internal excitation mechanisms in the magnetosphere (Takahashi and McPherron, 1984; Anderson et al., 1990; Ohtani et al., 1999), such as kinetic instabilities of ring current ions and high beta plasma clouds, and are more commonly radially polarized waves (e.g. Arthur and McPherron, 1981; Engebretson et al., 1992; Anderson, 1993). This asymmetry between morning end afternoon pulsations has been attributed to a greater stability of the afternoon magnetopause to shear-flow instabilities such as the Kelvin-Helmholtz instability with respect to the morning flank (Mann et al., 1999).

\subsection{On the distribution of pulsations in frequency}

There have been various observations of fluctuations that show simultaneous pulsations at various harmonics with a structured distribution of frequencies; this phenomenology has been theoretically described by the cavity mode model of Samson et al. (1991). Observations of multiple discrete frequencies have utilised radars, ground magnetometers, geosynchronous satellites and satellites in the solar wind, and report a superposition of frequencies 1.3, 1.9, 2.6, 3.4 , and $4.2 \mathrm{mHz}$, with small variations in the actual numbers between the various studies. At higher frequencies, Shimazu et al. (1995) have reported observations of Pc5 pulsations at frequencies of 3.3, 4.7, 5.9, and $7.1 \mathrm{mHz}$ simultaneously. Samson et al. (1992) and Walker et al. (1992) have reported that these frequencies vary little and do not depend on the geomagnetic condition. The statistical analysis of narrow-band events over a period of 8 years which was presented in Fig. 2 does not show any particular pattern in the distribution of the events as a function of frequency, however, it is possible that the statistical sample of events is not large enough to test whether a preference for certain frequencies is present in this data set. It should be noted that, although it is true that the use of the windowing method in the Dynamic Power Spectra as described in Sect. 2.1 acts as a high-pass filter, blocking 
pulsations of periods longer than the window length, for the 80-min long Hanning window used here, only pulsations of frequencies less than $0.4 \mathrm{mHz}$ cannot be detected, which is lower than the Pc5 range of pulsations that are being studied.

3.6 On the drift-resonant interaction of the pulsation with energetic electrons

From the theory of radial diffusion it is known that fluctuations in the magnetospheric electromagnetic fields on the timescale of the bounce-averaged drift period of energetic particles can violate their third adiabatic invariant; these fluctuations will cause a net radial motion in the particles' orbits that doesn't average to zero over a time much longer than their drift period (e.g. Chan et al., 1989; Elkington et al., 1999). However fluctuations in the magnetosphere are usually broad-band in nature, making the observation of such drift resonant interactions rather hard to directly detect. The phenomenon on 16-18 February gives the opportunity to monitor the interaction of a narrow-band fluctuation with energetic electrons of various drift frequencies around the frequency of the fluctuation. In order to explore the nature of this interaction, we performed a spectral analysis on the LANL energetic electron flux measurements, and plotted the corresponding dynamic spectra as a function of time. A similar method of treating flux data as field measurements, in the sense that a spectral analysis is performed on the particle count data to extract flux modulation in particular frequencies, was demonstrated by Lessard et al. (2003).

The spectral analysis has shown that, indeed, out of the four energy channels monitored, the third channel (shown in Fig. 6c), which includes electrons with drift frequencies within 2.9 to $4.9 \mathrm{mHz}$ that encompass the $4 \mathrm{mHz}$ narrowband fluctuation, shows the most intense flux modulation. The drift frequency range was calculated using $90^{\circ}$ pitch angle particles in a T96 magnetic field model (Tsyganenko, 1995). Even though drift frequency will vary with pitch angle, the pitch angle distributions at times of slightly disturbed magnetospheric conditions at the vicinity of geosynchronous orbit were found to be peaked at $90^{\circ}$ (Gannon et al., 2007); thus our calculations of the particles' drift frequencies are expected to be valid. As seen in Fig. 6c, flux modulation occurs in a single dominant frequency; in the other energy channels, more resonant frequencies appear. These include the frequency of the magnetospheric pulsation at $4 \mathrm{mHz}$, the drift frequency of the electrons in the energy channel, as well as harmonics and sub-harmonics of the narrow-band fluctuation. It should be emphasized that the region of intense fluctuations of the fluxes in Figs. 6a through 6d (red spots in the DPS) are only indicative of more intense flux modulation at the corresponding times and not changes in the average flux level. The flux magnitude remains relatively unchanged during and after the passage of the spacecraft through the region of intense fluctuations, as can be seen in the flux measurements, in the top panels of the four figures. Even though a clear drift resonance effect is observed, with electron flux modulation becoming more evident in the energy channels of electrons with drift frequencies closer to the magnetic fluctuation frequency, no net increase or decrease in energetic electron flux is observed, indicating that the net transport of electrons is not significant. We attribute this to the fact that the source population may not have a strong gradient in the phase space density, which is a necessary condition for enhanced radial diffusion and subsequent flux change to occur.

\section{Summary and conclusions}

A narrow-band Pc5 pulsation event was presented using geosynchronous measurements of the magnetic field by GOES satellites, ground magnetic field signatures by the CARISMA chain of ground magnetometers, LANL-SOPA energetic electrons, and outer magnetosphere magnetic field measurements from Geotail. The dominantly transverse pulsation occurs in the post-noon region. It is polarized mostly in the radial direction and does not compress the total magnetic field, leading us to believe that it is quasi-field-aligned, and more closely described as an Alfvén wave. Geotail was traversing the dayside outer magnetosphere near the equatorial plane in an ideal orbit that would allow the observation of solar wind-induced pulsations, if they were propagating earthward through the bow shock, magnetosheath and magnetopause. Therefore the waves observed at GOES are attributed to an internal generation mechanism, as there is no apparent connection to external pulsations originating in the solar wind during the time the pulsation was observed. However the exact generation mechanism could not be determined by the available set of measurements. LANL MPA plasma measurements that might show low-energy plasma bumps-on-tail with sufficient amounts of free energy, which could be the source of the pulsations, could be looked at in detail for this event. A comparison between the phases (cross-phase spectrogram) of GOES-8 and GOES-10 on 18 and 19 February (not shown), when both spacecraft were in the active region, shows no cross-correlation between the two signals. Taking into account the 4-h separation of the two s/c, this indicates that mode number $m$ should be higher than 6 . The lack of pulsation signatures on the ground also indicates that $m$ has most probably a high value.

The duration of the event that was studied is much longer than the theoretical estimates for the conversion of ideal compressional poloidal pulsations to toroidal and vise-versa, as well as estimates of the damping rates of pulsations. Thus it is speculated that the generation mechanism for the pulsations is acting over a prolonged time, and hence while perturbations are mode converted and/or dampened, new ones are produced. An expansion of the plasmasphere and the formation of related plasma tail or drainage plumes in the evening sector that are sustained over a 3-day period could explain the duration of the event. Another possible model was proposed 
recently by Ozeke and Mann (2001): it suggests that the narrow-band Pc5 pulsations in the afternoon sector are generated at the boundary between open and closed particle orbits. This boundary would have to remain stationary for the duration of the event, which is reasonable for the particularly quiet conditions in the magnetosphere during the time period of interest. However a detailed survey of the composition and energy distribution of the $\mathrm{eV}$ to tens-keV plasma is required in order to associate the fluctuations with a particular internal generation mechanism and/or a particular ion distribution function.

The statistical study of narrow-band compressional fluctuations at geosynchronous orbit that was presented in Fig. 2 revealed an asymmetry in the occurrence distribution, with an increased number of narrow-band fluctuation events in the post-noon/evening region. Other statistical studies of smaller samples have shown that pulsations in the post-noon and evening sector are more often related to internal excitation mechanisms, whereas morning and pre-noon pulsations are mostly related to external excitation mechanisms, and in particular Kelvin-Helmholtz-type instabilities. The individual event that was extensively studied was observed post-noon and the various analyses presented suggest that it was caused by an internal generation mechanism. The distribution of frequencies in the statistical analysis of the narrow-band events over the 8-year study does not show any particular pattern in the distribution of the events as a function of frequency. Thus the pulsations reported in the statistical study are most probably not related to observations that have reported simultaneous spectral peaks over a number of different frequencies that vary little in frequency between the various studies.

The pulsation event studied herein did not show a distinct narrow-band signature on the ground, even though there have been reports in the past of co-temporal measurements of fluctuation events with the same spectral characteristics in the magnetosphere and on the ground. It is understood that ground signatures are modified in a very complex way by both ionospheric and ground currents, and that ULF waves, in particular those of high mode number $\mathrm{m}$, may be altered or reflected along their propagation from the equatorial regions through the ionosphere to the ground; however a thorough statistical investigation of the narrow-band events in conjunction with a statistical investigation of ground signatures could reveal the exact conditions under which pulsations can penetrate to the ground.

Finally, a spectral analysis on energetic electron flux measurements has revealed that energetic electrons with drift frequencies close to the narrow-band pulsation frequency show a distinct flux modulation around the local time of the pulsation. A clear drift resonance effect is observed, with electron flux modulation becoming more intense in the energy channels of electrons with drift frequencies closer to the field fluctuation frequency. In addition, other modulations appear in harmonics and sub-harmonics of the electrons' drift frequency within this local time. However no significant net increase or decrease in energetic electron flux is observed. This has been attributed to a lack of sufficient gradient in the phase space density of the source population, which is a necessary condition for flux changes that are associated with radial diffusion.

Acknowledgements. This work was supported by NSF Grants (ATM-0519207, -0549093, and CISM) and by the "Pythagoras" 1249-4 grant. We thank both referees for their careful reading, their thoughtful comments and their suggestions.

Topical Editor I. A. Daglis thanks H. Luehr and another anonymous referee for their help in evaluating this paper.

\section{References}

Alfvén, H. and Falthammar, C. G.: Cosmical Electrodynamics, Oxford Univ. Press, New York, 1963.

Anderson, B. J., Engebretson, M. J., Rounds, S. P., Zanetti, L. J., and Potemra, T. A.: A statistical study of Pc 3-5 pulsations observed by the AMPTE/CCE magnetic field experiment, 1, Occurrence distributions, J. Geophys. Res., 95, 10 495-10 523, 1990.

Anderson, B. J.: Statistical studies of Pc 3-5 pulsations and their relevance for possible source mechanisms of ULF waves, Ann. Geophys., 11, 128-143, 1993, http://www.ann-geophys.net/11/128/1993/.

Arthur, C. W. and McPherron, R. L.: The statistical character of Pc 4 magnetic pulsations at synchronous orbit, J. Geophys. Res., 86, 1325-1334, 1981.

Bellan, P. M.: Mode conversion into non-MHD waves at the Alfvén layer: The case against the field line resonance concept, J. Geophys. Res., 101, $24887-24898,1996$.

Bellan, P. M.: New model for ULF Pc5 pulsations: Alfvén cones, Geophys. Res. Lett., 23, 1717-1720, 1996.

Belian, R. D., Gisler, G. R., Cayton, T., and Christensen, R.: High$\mathrm{Z}$ energetic particles at geostationary orbit during the Great Solar Proton Event series of October 1989, J. Geophys. Res., 97, 16897-16906, 1992

Chappell, C. R., Harris, K. K., and Sharp, G. W.: The morphology of the bulge region of the plasmasphere, J. Geophys. Res., 75, 3848-3861, 1970

Chan, A. A., Chen, L., and White, R. B.: Interaction of ring current protons with Pc 5 waves, Geophys. Res. Lett., 16, 1133-1136, 1989.

Chen, L. and Hasegawa, A.: A theory of long-period magnetic pulsations, 1., Steady state excitation of field line resonances, J. Geophys. Res., 79, 1024-1032, 1974.

Chen, A. J., Grebowsky, J. M., and Marubashi, K.: Diurnal variation of thermal plasma in the plasmasphere, Planet. Space Sci., 24 765-769, 1976.

Cowley, S. W. H. and Ashour-Abdalla, M.: Adiabatic plasma convection in a dipole field: Proton forbidden-zone effects for a simple electric field model, Planet. Space Sci., 24, 821-833, 1976.

Denton, R. E., Lessard, M. R., and Kistler, L. M.: Radial localization of magnetospheric guided poloidal Pc 4-5 waves, J. Geophys. Res., 108, 1105, doi:10.1029/2002JA009679, 2003.

Dungey, J. W.: Electrodynamics of the Outer Atmosphere, Ionos. Res. Lab. Sci. Rept., 69. Pennsylvania State University, 1954.

Elkington, S. R., Hudson, M. K., and Chan, A. A.: Acceleration of relativistic electrons via drift-resonant interaction with toroidal- 
mode Pc5 ULF oscillations, Geophys. Res. Lett., 26, 3273-3276, 1999.

Engebretson, M. J., Murr, D. L., Erickson, K. N., Strangeway, R. J., Klumpar, D. M., Fuselier, S. A., Zanetti, L. J., and Potemra, T. A.: The spatial extent of radial magnetic pulsation events observed in the dayside near synchronous orbit, J. Geophys. Res., 97, 13741-13 758, 1992.

Falthammar, C. G.: Radial diffusion by violation of the third adiabatic invariant, in Earth's Particles and Fields, Reinhold, New York, p. 157, 1968.

Francia, P. and Vilante, U.: Some evidence of ground power enhancements at frequencies of global magnetospheric modes at low latitudes, Ann. Geophys., 15, 17-23, 1997,

http://www.ann-geophys.net/15/17/1997/.

Fujita, S. and Glassmeier, K.-H.: Magnetospheric cavity resonance oscillations with energy flow across the magnetopause, J. Geomag. Geoelectr., 47, 1277-1292, 1995.

Gannon, J. L., Li, X., and Heynderickx, D.: Pitch angle distribution analysis of radiation belt electrons based on Combined Release and Radiation Effects Satellite Medium Electrons A data, J. Geophys. Res., 112, A05212, doi:10.1029/2005JA011565, 2007.

Green, J. C. and Kivelson, M. G.: Relativistic electrons in the outer radiation belt: Differentiating between acceleration mechanisms, J. Geophys. Res., 109, A03213, doi:10.1029/2003JA010153, 2004.

Hasegawa, A.: Drift mirror instability in the magnetosphere, Phys. Fluids, 12, 2642-2650, 1969.

Jacobs, J. A., Kato, Y., Matsushita, S., and Troitskaya, V. A.: Classification of geomagnetic micropulsations, J. Geophys. Res., 69, 180-181, 1964.

Hughes, W. J. and Southwood, D. J.: The screening of micropulsation signals by the atmosphere and ionosphere, J. Geophys. Res., 81, 3234-3240, 1976.

Hughes, W. J., Southwood, D. J., Mauk, B., McPherron, R. L., Barfield, J. N.: Alfvén waves generated by an inverted plasma energy distribution, Nature, 275, 43-45, 1978.

Kepko, L., Spence, H. E., and Singer, H.: ULF waves in the solar wind as direct drivers of magnetospheric pulsations, Geophys. Res. Lett., 29, 1197, doi:10.1029/2001GL014405, 2002.

Kivelson, M. G. and Southwood, D. J.: Resonant ULF waves: A new interpretation, Geophys. Res. Lett., 12, 49-52, 1985.

Kivelson, M. G. and Chen, S.-H.: The magnetopause: Surface waves and instabilities and their possible dynamic consequences, in: Physics of the Magnetopause, Geophys. Monogr. Ser., vol. 90, edited by: Song, P., Sonnerup, B. U. O., and Thomsen, M. F., p. 257, AGU, Washington D.C., 1995.

Kokubun, S., Kivelson, M. G., McPherron, R. L., and Russel, C. T.: OGO 5 observations of Pc 5 waves: Particle flux modulations, J. Geophys. Res., 82, 2774-2786, 1977.

Lanzerotti, L. J., Hasegawa, A., and Maclennan, C. G.: Drift mirror instability in the magnetosphere: Particle and field oscillations and electron heating, J. Geophys. Res., 74, 5565-5578, 1969.

Lanzerotti, L. J., Maclennan, C. G., Fukunishi, H., Walker, J. K., and Williams, D. J.: Latitude and longitude dependence of storm time Pc5 type plasma wave, J. Geophys. Res., 80, 1014-1018, 1975.

Lee, D. H.: Dynamics of MHD wave propagation in the low-latitude magnetosphere, J. Geophys. Res., 101, 15 371-15 386, 1996.

Lessard, M. R., Hanna, J., Donovan, E. F., and Reeves, G.
D.: Evidence for a discrete spectrum of persistent magnetospheric fluctuations below $1 \mathrm{mHz}$, J. Geophys. Res., 108, 1125, doi:10.1029/2002JA009311, 2003.

Li, X., Baker, D. N., O’Brien, T. P., Xie, L., and Zong, Q. G.: Correlation between the inner edge of outer radiation belt electrons and the innermost plasmapause location, Geophys. Res. Lett., 33, L14107, doi:10.1029/2006GL026294.

Mann, I. R. and Wright, A. N.: Finite lifetimes of ideal poloidal Alfvén waves, J. Geophys. Res., 100, 23 677-23 686, 1995.

Mann, I. R., Wright, A. N., Mills, K. J., and Nakariakov, V. M.: Excitation of magnetospheric waveguide modes by magnetosheath flows, J. Geophys. Res., 104, 333-353, 1999.

McPherron, R. L., Russell, C. T., and Coleman Jr., P. J.: Fluctuating magnetic fields in the magnetosphere, 2, ULF waves, Space Sci. Rev., 13, 411-454, 1972.

Misra, K. D., Nielsen, E., and Lühr, L.: STARE and SABRE observations of a Pc5 pulsation with large azimuthal wave number during a storm period, J. Geophys. Res., 90, 10 923-10 934, 1985.

Newton, R. S., Southwood, D. J., and Hughes, W.: Damping of geomagnetic pulsations by the ionosphere, Planet. Space Sci., 26, 201-209, 1978.

Ohtani, S.-I., Rostoker, G., Takahashi, K., Angelopoulos, V., Nakamura, M., et al.: Coordinated ISTP satellite and ground observations of morningside Pc5 waves, J. Geophys. Res., 104, 23812397, 1999.

Onsager, T. G., Chan, A. A., Fei, Y., Elkington, S. R., Green, J. C., and Singer, H. J.: The radial gradient of relativistic electrons at geosynchronous orbit, J. Geophys. Res., 109, A05221, doi:10.1029/2003JA010368, 2004.

Ozeke, L. G. and Mann, I. R.: Modeling the properties of high $\mathrm{m}$ Alfvén waves driven by the drift-bounce resonance mechanism, J. Geophys. Res., 106, 15 583-15 598, 2001.

Reeves, G. D., Belian, R. D., Cayton, T. C., Henderson, M. G., Christensen, R. A., McLachlan, P. S., and Ingraham, J. C.: Using Los Alamos geosynchronous energetic particle data in support of other missions, in: Satellite-Ground Based Coordination Source Book, edited by: Lockwood, M., Opgenoorth, H. J., Wild, M. N., and Stampe, R., pp. 263-272, Eur. Space Agency, Paris, 1997.

Rioul, O. and Vetterli, M.: Wavelets and signal processing, IEEE Signal Processing Magazine, 8(4), 14-38, 1991.

Ruohoniemi, J. M., Greenwald, R. A., Baker, K. B., and Samson, J. C.: HF radar observations of Pc 5 field line resonances in the midnight early morning MLT sector, J. Geophys. Res., 96, 15 697-15710, 1991.

Samson, J. C., Greenwald, R. A., Ruohoniemi, J. M., Hughes, T. J., and Wallis, D. D.: Magnetometer and radar observations of magnetospheric cavity modes in the Earths magnetosphere, Can. J. Phys., 69, 929-937, 1991.

Samson, J. C., Harrold, B. G., Ruohoniemi, J. M., Greenwald, R. A., and Walker, A. D. M.: Field line resonances associated with MHD waveguides in the magnetosphere, Geophys. Res. Lett., 19, 441-444, 1992.

Sarafopoulos, D. V.: Long duration Pc 5 compressional pulsations inside the Earths magnetotail lobes, Ann. Geophys., 13, 926937, 1995, http://www.ann-geophys.net/13/926/1995/.

Schulz, M. and Lanzerotti, L. J.: Particle diffusion in the radiation belts, Springer, New York, 1974. 
Shimazu, H., Araki, T., Kamei, T., and Hanado, H.: A symmetric appearance of Pc5 on dawn and dusk side associated with solar wind dynamic pressure enhancement, J. Geomag. Geoelectr., 47, 177-189, 1995.

Singer, H. J. and Kivelson, M. G.: The Latitudinal distribution of Pc5 waves in space: magnetic and electric field observations, J. Geophys. Res., 84, 7213-7222, 1979.

Singer, H. J., Hughes, W. J., and Russell, C. T.: Standing Hydromagnetic waves observed by ISEE 1 and 2: Radial extent and harmonic, J. Geophys. Res., 87, 3519-3527, 1982.

Singer, H. J., Matheson, L., Grubb, R., Newman, A., and Bouwer, S. D.: Monitoring space weather with GOES Magnetometers, SPIE Proc., 2812, 1996.

Southwood, D. J., Dungey, J. W., and Eherington, R. L.: Bounce resonant interaction between pulsations and trapped particles, Planet. Space Sci., 17, 349-361, 1969.

Southwood, D. J.: Some features of field line resonances in the magnetosphere, Planet. Space Sci., 22, 483-491, 1974.

Takahashi, K. and McPherron, R. L.: Standing hydromagnetic oscillations in the magnetosphere, Planet. Space Sci., 32, 13431359, 1984.

Takahashi, K., Higbie, P. R., and Baker, D. N.: Azimuthal propagation and frequency characteristic of compressional Pc 5 waves observed at geostationary orbit, J. Geophys. Res., 1473-1485, 1985a.

Takahashi, K., Russell, C. T., and Anderson, R. R.: ISEE 1 and 2 observation of the spatial structure of a compressional Pc 5 wave, Geophys. Res. Lett., 12, 613-616, 1985 b.

Takahashi, K., Sato, N., Warnecke, J., Lühr, H., Spence, H. E., and Tonewaga, Y.: On the standing wave mode of giant pulsations, L. Geophys. Res., 97, 10 717-10 732, 1992.
Takahashi, K.: ULF waves: 1997 IAGA division 3 reporter review, Ann. Geophys., 16, 787-803, 1998, http://www.ann-geophys.net/16/787/1998/.

Tepley, L. R. and Landshoff, R. K.: Waveguide theory for ionospheric propa gation of hydromagnetic emissions J. Geophys. Res., 71, 1499-1504, 1966.

Tsyganenko, N. A.: Modeling the Earth's magnetospheric magnetic field confined within a realistic magnetopause, J. Geophys. Res., 100, 5599-5612, 1995.

Vetoulis, G. and Chen, L.: Global structures of Alfvén -ballooning modes in magnetospheric plasmas, Geophys. Res. Lett., 21, 2091-2094, 1994.

Vetoulis, G. and Chen, L.: Kinetic theory of geomagnetic pulsations, 3, Global analysis of drift Alfvén ballooning modes, J. Geophys. Res., 101, 15 441-15 456, 1996.

Walker, A. D. M., Ruohoniemi, J. M., Baker, K. B., Greenwald, R. A., and Samson, J. C.: Spatial and temporal behaviour of ULF pulsations observed by the Goose Bay HF radar, J. Geophys. Res., 97, 12 187-12 202, 1992.

Waters, C. L., Samson, J. C., and Donovan, E. F.: Variation of plasmatrough density derived from magnetospheric field line resonances, J. Geophys. Res., 101, 24 737-24 745, 1996.

Wright, A. N. and Allan, W.: Structure, phase motion, and heating within Alfvén resonances, J. Geophys. Res., 101, 17 399-17408, 1996.

Wright, A. N. and Rickard, G. J.: A numerical study of resonant absorption in a magnetohydrodynamic cavity driven by a broadband spectrum, Astrophys. J., 444, 458-470, 1995.

Wu, C. C.: Kelvin-Helmholtz instability at the magnetopause boundary, J. Geophys. Res., 91, 3042-3060, 1986. 